\title{
Numerical study of the phase slip in two-dimensional superconducting strips
}

\author{
Chunyin Qiu and Tiezheng Qian* \\ Department of Mathematics, Hong Kong University of Science and Technology, Clear Water Bay, Kowloon, Hong Kong
}

(Received 27 February 2008; revised manuscript received 28 April 2008; published 30 May 2008; publisher error corrected 10 June 2008 )

\begin{abstract}
In this paper, we numerically investigate the phase slips in two-dimensional (2D) superconducting strips using the string method, which has been presented as an efficient tool for the study of thermally activated rare events. In the framework of Ginzburg-Landau (GL) theory, we calculate the most probable transition pathway for thermally activated phase slips that are responsible for spontaneous current dissipation. Along the most probable pathway, the saddle point of the GL free-energy functional can be located, from which the energy barrier is also determined. We find there exists a critical width $w_{c}$ for narrow strips. Below $w_{c}$, the strip behaves as a one-dimensional superconducting wire for which the phase slips are described by the LangerAmbegaokar-McCumber-Halperin theory [Phys. Rev. 164, 498 (1967); Phys. Rev. B 1, 1054 (1970)]. Above $w_{c}$, however, the $2 \mathrm{D}$ character of the strip is recovered, and the phase slips are dominated by vortices crossing the strip. In this 2D regime, our numerical results based on the GL theory are compared to the analytical results of the London theory. While there is good agreement for large strip widths, deviation is noticed for small widths $\left(\right.$ still $>w_{c}$ ) because of the closeness of vortex core to the strip edges.
\end{abstract}

DOI: 10.1103/PhysRevB.77.174517 PACS number(s): 74.40.+k, 74.20.De, 82.20.Wt, 05.10. $-\mathrm{a}$

\section{INTRODUCTION}

It has been known for decades that, in one-dimensional (1D) superconducting systems, i.e., wires, thermally activated phase slips are responsible for the dissipation of supercurrent at temperatures below the critical temperature $T_{c} .{ }^{1-3}$ The Langer-Ambegaokar-McCumber-Halperin (LAMH) theory ${ }^{2,3}$ provides a theoretical framework for quantitative studies of the resistance of superconducting wires below $T_{c}{ }^{4}$ In two-dimensional (2D) superconducting thin films that are wide enough, the current dissipation is due to the flow of free vortices induced by the thermally activated (and/or current assisted) unbinding of vortex-antivortex pairs (VAPs), quantitatively described by the resistance theory based on the Kosterlitz-Thouless transition. ${ }^{5-11}$ The above 1D and 2D cases have been extensively investigated both theoretically and experimentally. ${ }^{1-11}$ However, the case of quasi-2D narrow superconducting strips has received relatively less attention. ${ }^{12-18}$ The investigation on this intermediate dimension is important as well considering its relevance to some superconducting detecting devices, e.g., superconducting single-photon detectors ${ }^{12-17}$ and superconducting submillimeter detectors. ${ }^{18}$ In particular, the mechanism of current dissipation in narrow strips remains an issue. ${ }^{12-18}$ Interpretations of experimental data for these systems involve two distinct mechanisms. While in Refs. 14 and 15, the strips are treated as 2D films in which the current dissipation is attributed to the thermally activated unbinding of VAPs, the competition between 1D phase-slip strips and 2D vortices has been modeled in Refs. 16 and 17 for quasi-2D superconductors. In this paper, we show that, for narrow superconducting strips, typically of a small width $w \sim 10 \xi{ }^{15-17}$ where $\xi$ is the temperature-dependent correlation length, the current dissipation is mostly caused by (discrete) phase slips, each accomplished by a single thermally activated vortex crossing the strip.

Compared to the $1 \mathrm{D}$ case, the complexity of 2D superconductors arises from the existence of topological defects, i.e., vortices. A simplified description based on the London theory has been used to obtain certain analytical solutions, in which the vortex is treated as a point particle by neglecting the core structure within a radius $\sim \xi .{ }^{19}$ When applied to problems of current distribution and magnetic moment in stable and metastable states, the London theory can always yield a good agreement with the more complete GinzburgLandau (GL) theory. In the problem of thermally activated barrier crossing, however, the core energy has to be explicitly taken into account, especially for narrow strips carrying a strong current. Therefore, a full treatment based on the GL theory is necessitated plus an efficient numerical tool to deal with the GL free-energy functional. Mathematically, in a 2D GL description of narrow strips, it would be very difficult to obtain any analytical solution for the saddle point of freeenergy functional and the corresponding free-energy barrier.

Recently, the string method ${ }^{20-22}$ has been presented for the numerical evaluation of thermally activated rare events. This method first locates the most probable transition pathway connecting two stable and/or metastable states in configuration space. This is done by evolving strings, which are smooth curves with intrinsic parametrization in configuration space, toward the minimal energy path (MEP). We have demonstrated the string method to be an efficient numerical tool for evaluating the thermally activated phase slips in 1D superconducting wires. ${ }^{23,24}$ The purpose of this paper is to investigate the thermally activated phase slips in quasi-2D narrow superconducting strips using the string method.

The paper is organized as follows. To make the problem under investigation clear, some basic considerations and assumptions are presented in Sec. II. The theoretical model is presented in Sec. III followed by the numerical results in Sec. IV. The paper is concluded in Sec. V with a brief summary of the main results.

\section{BASIC CONSIDERATIONS AND ASSUMPTIONS}

In the absence of external magnetic field, the current dissipation in 2D superconducting films is due to the thermally 
activated (and/or current assisted) flow of free vortices, which are either generated in the bulk (from unbounded VAPs) or nucleated at the edges, with the corresponding generation rates (defined as the number of free vortices generated per unit time) given by $\Gamma_{b}=\Omega_{b} e^{-\Delta F_{b} / k_{B} T}$ and $\Gamma_{e}$ $=\Omega_{e} e^{-\Delta F_{e} / k_{B} T}$, respectively. Here, $\Omega_{b(e)}$ denotes the prefactor and $\Delta F_{b(e)}$ denotes the energy barrier for the bulk (edge) contribution, $k_{B}$ is the Boltzmann constant, and $T$ is the temperature. Physically, the entropic effect is included in the prefactor, for which we have $\Omega_{b} \propto A / \xi^{2}$ and $\Omega_{e} \propto l / \xi$ based on the consideration of translational symmetry, where $A$ and $l$ are the sample area and length (along the current direction). As for the energy barriers, we have $\Delta F_{b} \cong 2 \Delta F_{e}{ }^{25}$ A competition arises from the above relations: the bulk contribution is enhanced by the prefactor but suppressed by the energy barrier while the edge contribution displays the opposite trend. For wide films, the sample area is large enough to make $\Gamma_{b}$ dominate in the generation of free vortices. For narrow strips, however, the edge contribution $\Gamma_{e}$ becomes dominant because of the lower energy barrier. ${ }^{25}$ It is rather involved to determine the characteristic dimension for the crossover from (wide) films to (narrow) strips because many physical parameters are involved, including the sample size, temperature, external current, and a few material properties. In this paper, we simply assume that the strips are narrow enough for us to ignore the contribution of free vortices generated from unbounded VAPs in the bulk. We focus on narrow strips of width $w \sim 10 \xi$, for which numerical calculations show that the current dissipation is caused by phase slips of $2 \mathrm{D}$ character, each realized through a single thermally activated vortex crossing the strip.

Free vortices crossing the strip are controlled by two time scales. The first is associated with the thermally activated barrier crossing, $\tau_{1}=1 / \Gamma_{e}$. The second is associated with a free vortex crossing the strip, $\tau_{2}=w / v$, where $v$ is the average velocity of vortex movement and $w$ the strip width. ${ }^{26,27}$ If $\tau_{1} \gg \tau_{2}$, then phase slips occur as well-separated discrete events. On the contrary, if $\tau_{2} \gg \tau_{1}$, then the numerous coexisting vortices (with the average number approximated by $N_{F} \cong \tau_{2} / \tau_{1} \gg 1$ ) lead to a continuous sequence of phase slips. For wide films, the latter is always assumed. ${ }^{5-11}$ In order to take into account the intervortex screening effects, the renormalization group technique is used in developing the resistance theory based on the Kosterlitz-Thouless transition. However, for the narrow strips considered in this paper, the assumption of $\tau_{2} \gg \tau_{1}$ is no longer justified. In fact, from $\tau_{1}$ $=1 / \Gamma_{e} \sim e^{\Delta F_{e} / k_{B} T}$ and $\tau_{2} \propto w$, we expect $\tau_{1} \gg \tau_{2}$ if $\Delta F_{e} \gg k_{B} T$ and the strip is sufficiently narrow. Therefore, it is extremely improbable for several vortices to coexist in a narrow strip. For this reason, hereafter, we ignore the intervortex screening effect and consider only discrete phase slips, each realized through a single vortex crossing the strip.

The voltage-current $(V-I)$ characteristic is always an important issue, irrespective of system dimension. Because of the discrete nature of phase slips, the resistance expression for narrow strips has to be distinguished from that for wide films ${ }^{8,11}$ but forms a good analog to the LAMH expression for $1 \mathrm{D}$ wires in which the current dissipation is caused by current-biased barrier crossing. A natural result of the LAMH theory is the linearity of $V-I$ characteristic for weak

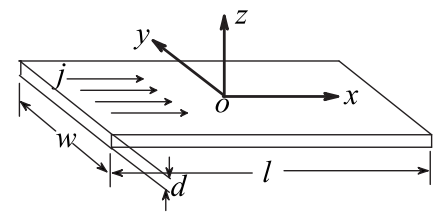

FIG. 1. A schematic illustration of a superconducting strip and the coordinate system. The length, width, and thickness of the strip are $l, w$, and $d$. The current flows along the $x$ direction.

currents. $^{2}$ [This linearity has a very simple origin. The difference in free energy between neighboring states is proportional to the current. Therefore, to the leading order, the difference in transition rate between upward and downward transitions is also proportional to the current. The voltage is linearly related to this rate difference.] We note that the linear $V-I$ characteristic has been reported for narrow strips in some literatures. ${ }^{15-17}$

In this paper, we will focus on the narrow strips of width $w \sim 10 \xi$ and investigate the thermally activated phase slips responsible for current dissipation. The typical width considered here is actually close to the characteristic dimension for the crossover from 2D strip to 1D wire. Physically, there exists a critical width below which the strip is effectively $1 \mathrm{D}$ and so the 2D-like phase slips realized through vortex motion across the strip can no longer be activated. However, the 1D-like phase slips described by the LAMH theory may always contribute to the current dissipation in a narrow strip. Therefore, above the critical width, a competition appears between the 1D-like phase slips (with no vortex involved) and the 2D-like phase slips involving vortices, and the dominant contribution is to be determined by comparing their respective energy barriers.

\section{PHASE-SLIP FLUCTUATIONS IN NARROW SUPERCONDUCTING STRIPS}

\section{A. Superconducting strips}

Figure 1 shows a superconducting strip carrying a current. The strip is parallel to the $x y$ plane and the current flows along the $x$ direction. The origin of the coordinate system is set at the center of strip. The length, width, and thickness of the strip are denoted by $l, w$, and $d$, respectively. For thin strips, the thickness $d$ is much smaller than the temperaturedependent London penetration length $\lambda$, and the superconducting behavior in the $x y$ plane is characterized by an effective penetration length $\Lambda=2 \lambda^{2} / d$ that can be much larger than the temperature-dependent correlation length $\xi$, even for type I superconductors. ${ }^{28}$ In this paper, the strip width $w$ is assumed to be much smaller than $\Lambda$, and hence the selfinduced magnetic field can be neglected. In the absence of applied magnetic field, the 2D GL free-energy functional is of the form

$$
F[\psi(x, y)]=d \int d x d y\left[\frac{K}{2}|\nabla \psi|^{2}-\frac{\alpha}{2}|\psi|^{2}+\frac{\beta}{4}|\psi|^{4}\right],
$$

where $K=\hbar^{2} / m, \hbar$ is the Planck constant and $m$ is the effective mass of a Cooper pair, $\alpha=\alpha_{0}\left(T_{c}-T\right)$, and $\alpha_{0}$ and $\beta$ are 
both phenomenological material parameters. The time evolution of $\psi$ is governed by the time-dependent GL equation with a white noise,

$$
\gamma \frac{\partial \psi}{\partial t}=-\frac{1}{d} \frac{\delta F[\psi]}{\delta \psi^{*}}+\zeta=K \nabla^{2} \psi+\alpha \psi-\beta|\psi|^{2} \psi+\zeta,
$$

where $\gamma$ is the damping coefficient, the asterisk denotes the complex conjugate, and $\zeta(x, y, t)$ is a white noise satisfying the autocorrelation functions,

$$
\begin{gathered}
\left\langle\zeta(x, y, t) \zeta\left(x^{\prime}, y^{\prime}, t^{\prime}\right)\right\rangle=0, \\
\left\langle\zeta(x, y, t) \zeta^{*}\left(x^{\prime}, y^{\prime}, t^{\prime}\right)\right\rangle=4 d^{-1} \gamma k_{B} T \delta\left(x-x^{\prime}\right) \delta\left(y-y^{\prime}\right) \delta\left(t-t^{\prime}\right) .
\end{gathered}
$$

This noise generates a random motion of $\psi(x, y)$ in the configuration space and stabilizes an equilibrium distribution with the probability density proportional to $e^{-F[\psi] / k_{B} T}$.

For computational purpose, we use the dimensionless form

$$
\bar{F}[\bar{\psi}(\bar{x}, \bar{y})]=\int d \bar{x} d \bar{y}\left[\frac{1}{2}|\bar{\nabla} \bar{\psi}|^{2}-\frac{1}{2}|\bar{\psi}|^{2}+\frac{1}{4}|\bar{\psi}|^{4}\right]
$$

for the free-energy functional. Here, the overbar denotes the dimensionless quantities, obtained with $F$ scaled by $G(T)$ $=d \xi^{2} \alpha^{2} / \beta, \psi$ by $\sqrt{\alpha / \beta}$, and $x$ and $y$ by the temperaturedependent correlation length $\xi=\sqrt{K / \alpha}$. Here, $\alpha^{2} / 4 \beta$ $=H_{c}^{2}(T) / 8 \pi$ is the bulk condensation energy density, with $H_{c}(T)$ being the bulk critical field. The corresponding dimensionless time-dependent GL equation is of the form

$$
\frac{\partial \bar{\psi}}{\partial \bar{t}}=-\frac{\delta \bar{F}[\bar{\psi}]}{\delta \bar{\psi}^{*}}+\bar{\zeta}=\bar{\nabla}^{2} \bar{\psi}+\left(1-|\bar{\psi}|^{2}\right) \bar{\psi}+\bar{\zeta},
$$

in which the time is scaled by $\tau(T)=\gamma / \alpha$, i.e., $\bar{t}=t / \tau(T)$, and the dimensionless noise $\bar{\zeta}$ satisfies the autocorrelation functions

$$
\begin{gathered}
\left\langle\bar{\zeta}(\bar{x}, \bar{y}, \bar{t}) \bar{\zeta}\left(\bar{x}^{\prime}, \bar{y}^{\prime}, \bar{t}^{\prime}\right)\right\rangle=0, \\
\left\langle\bar{\zeta}(\bar{x}, \bar{y}, \bar{t}) \bar{\zeta}^{*}\left(\bar{x}^{\prime}, \bar{y}^{\prime}, \bar{t}^{\prime}\right)\right\rangle \\
=4\left[k_{B} T / G(T)\right] \delta\left(\bar{x}-\bar{x}^{\prime}\right) \delta\left(\bar{y}-\bar{y}^{\prime}\right) \delta\left(\bar{t}-\bar{t}^{\prime}\right) .
\end{gathered}
$$

Throughout the remainder of this paper, we will mostly use the dimensionless quantities with the overbar dropped. We want to point out that all the temperature effects have been absorbed into the units (e.g., $G(T), \sqrt{\alpha / \beta}$, and $\xi$ ) in defining the dimensionless quantities.

\section{B. Metastable states, saddle points, and phase slips}

The metastable current-carrying states are the local minima of the GL free-energy functional, which can be obtained from the stationary GL equation

$$
\nabla^{2} \psi+\left(1-|\psi|^{2}\right) \psi=0
$$

It is convenient to take $\psi(x, y)=f(x, y) e^{i \varphi(x, y)}$, in which $f$ and $\phi$ represent the magnitude and phase of the complex order parameter. Then, Eq. (5) becomes

$$
\Delta f-f \nabla \phi \cdot \nabla \phi+\left(1-f^{2}\right) f=0
$$

and

$$
2 \nabla \phi \cdot \nabla f+f \Delta \phi=0,
$$

which should be supplemented by appropriate boundary conditions. The periodical boundary condition $\psi(-l / 2, y)$ $=\psi(l / 2, y)$ is imposed along the $x$ direction, and the superconductor-insulator boundary condition is applied at the two edges $y= \pm w / 2$, i.e., $\psi_{y}(x, \pm w / 2)=0$ (with the subscript $y$ denoting the partial derivative with respect to $y$ ). Under these boundary conditions, the metastable states are of uniform magnitude, i.e., $\nabla f=0$, and the solutions of Eqs. (6a) and $(6 b)$ are of the form

$$
\psi_{n}(x, y)=f_{n} e^{i k_{n} x},
$$

where $k_{n}=2 n \pi / l$ is the wave vector along the $x$ direction, $f_{n}=\sqrt{1-k_{n}^{2}}$ is the constant magnitude, and $n$ is an integer usually called the winding number. The (dimensionless) current density (along $x$ ) in the state of $\psi_{n}$ is $j_{n}=f_{n}^{2} k_{n}$ $=\left(1-k_{n}^{2}\right) k_{n}$. For $\psi_{n}$ to be metastable, $\left|k_{n}\right|$ must not exceed the critical wave vector $k_{c}=1 / \sqrt{3}$, which corresponds to the critical current density $j_{c}=\sqrt{4 / 27}$.

Between two neighboring metastable states $\psi_{n}$ and $\psi_{n-1}$, there is a saddle point of the GL free-energy functional, $\psi_{s}(x, y)$, which is also a solution of the stationary GL equation (5). Physically, this saddle point corresponds to the most probable thermally activated fluctuation which can carry the system from $\psi_{n}$ to $\psi_{n-1}$ and vice versa. The free-energy barrier $\Delta F$ is given by $\Delta F=F_{s}-F_{n}$ (or $\Delta F=F_{s}-F_{n-1}$ ) for the transition $\psi_{n} \rightarrow \psi_{n-1}$ (or $\psi_{n-1} \rightarrow \psi_{n}$ ), with $F_{n}$ (or $F_{n-1}$ ) and $F_{s}$ denoting the free energies of $\psi_{n}\left(\right.$ or $\left.\psi_{n-1}\right)$ and $\psi_{s}$, respectively. According to the free-energy expression $F_{n}=-\left(1-k_{n}^{2}\right)^{2} w l / 4$, a smaller winding number (corresponding to a weaker current) yields a lower free energy. Therefore, the transition $\psi_{n} \rightarrow \psi_{n-1}$ (for positive $n$ ) with a phase change by $-2 \pi$ (over the system length $l$ ) is much more probable than the transition $\psi_{n-1} \rightarrow \psi_{n}$ with a phase change by $2 \pi$ since the free-energy barrier $\Delta F=F_{s}-F_{n}$ is smaller. Because of this current-biased barrier crossing, the thermally activated phase slips result in a spontaneous process in which the free energy is lowered and the current is reduced. Based on this observation and also for the convenience of presentation, our discussions below will be focused on the currentreducing transition $\psi_{n} \rightarrow \psi_{n-1}$; the extension to the transition $\psi_{n-1} \rightarrow \psi_{n}$ is straightforward.

Physically, there exist two distinct kinds of phase slips corresponding to two distinct kinds of saddle points. Phase slips of the first kind are numerically obtained when the strip acts as a 1D wire, with the order parameter uniformly distributed along the $y$ direction across the strip. The corresponding saddle point will be called the phase-slip-strip (PSS) solution ${ }^{16}$ and denoted by $\psi_{s}^{\text {PSS }}$. The $1 \mathrm{D}$ character of phase slips of the first kind means that the PSS solution $\psi_{s}^{P S S}$ and the corresponding free-energy barrier $\Delta F^{\mathrm{PSS}}$ are quantitatively described by the LAMH theory. With the strip acting as a wire, the free-energy barrier $\Delta F^{\mathrm{PSS}}$ is proportional to the strip width $w$. The numerical evidence will be shown in Sec. IV. 
Phase slips of the second kind are more complicated because they involve vortices and thus $2 \mathrm{D}$ variations of the order parameter. A typical phase slip of the second kind will be shown in Sec. IV. The corresponding saddle point will be called the phase-slip-vortex (PSV) solution and denoted by $\psi_{s}^{\mathrm{PSV}}$. In the approximate description based on the London theory, the internal structure of the vortex core is neglected and the PSV solution is just represented by a position of the vortex center that yields the maximum free energy (see Appendix A for details). One advantage of this description is that analytical expressions can be obtained for the saddle point from the position of vortex center to the value of freeenergy barrier. However, in the framework of GL theory, analytical solution for $\psi_{s}^{\mathrm{PSV}}$ is no longer available, and hence a numerical approach becomes imperative. The string method has already been demonstrated to be an efficient numerical tool for the evaluation of thermally activated phase slips in 1D superconducting wires. ${ }^{23,24}$ In this paper, we will extend this numerical approach to investigate the PSV solution $\psi_{s}^{\mathrm{PSV}}$ and the corresponding free-energy barrier $\Delta F^{\mathrm{PSV}}$ in quasi-2D narrow superconducting strips.

\section{NUMERICAL RESULTS}

\section{A. Phase slips via single vortex crossing the strip}

Now, we present the numerical details for the evaluation of saddle points that intervene neighboring metastable states and result in phase slips. Without losing generality, we start from the transition $\psi_{3} \rightarrow \psi_{2}$ for a system of length $l=200$ and width $w=16$. The string method (outlined in Appendix B) is implemented as follows. Any configuration $\psi(x, y)$ is represented by a column vector of $2 N \times M$ entries (where 2 comes from the complex nature of $\psi$ ), with the $2 \mathrm{D}$ space $[-l / 2, l / 2] \times[-w / 2, w / 2]$ discretized by a uniform mesh of $N \times M=800 \times 64$ points. Spatial derivatives in the potential gradient $-\delta F[\psi] / \delta \psi^{*}$ are evaluated using the central finite difference. Discretized by $K=101$ points in the configuration space of $\psi(x, y)$, the string is evolved toward its stationary state which gives the MEP corresponding to the most probable transition pathway. During the string evolution, a reparametrization by arc length is applied every ten steps. The saddle point (either PSS or PSV) is a point that yields the maximum free energy along the MEP. Once the stationary MEP is obtained, the climbing image technique can be combined with the string method to locate the saddle point accurately. ${ }^{22,29}$ To check the uniqueness of the MEP, different initial strings have been tried. The simplest initial string is constructed by a linear interpolation between the metastable states $\psi_{3}$ and $\psi_{2}$ [expressed in Eq. (7)] and leads to the PSS MEP (i.e., the MEP along which the saddle point is found to be the PSS solution). Mathematically, a linear interpolation between $\psi_{3}$ and $\psi_{2}$ involves only 1D variation of $\psi$ along the $x$ direction and so does the saddle point $\psi_{s}^{\mathrm{PSS}}$ obtained from such an initial string. In order to obtain the PSV MEP (i.e., the MEP along which the PSV solution occurs at the saddle point), a small perturbation to the above initial string has to be used to introduce some variation along the $y$ direction as well.

Figure 2(a) shows the free-energy variation along the PSV
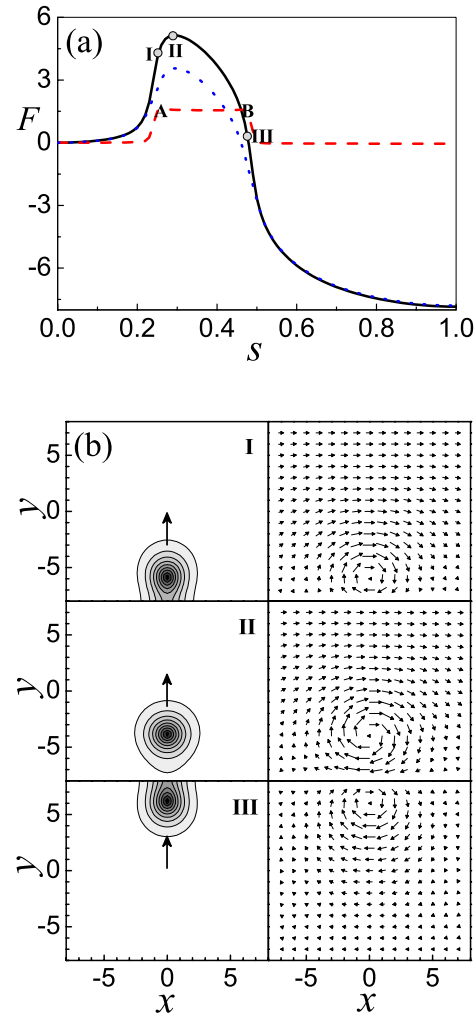

FIG. 2. (Color online) (a) Free energy (solid line) and the two of its ingredients, i.e., condensation energy (dashed line) and kinetic energy (dotted line), evaluated along the PSV MEP from $\psi_{3}$ to $\psi_{2}$, each plotted as a function of the arc length $s$ in the $\psi(x, y)$-function space, for $l=200$ and $w=16$. The $\psi_{3}$ state is taken as the reference point at which $s=0$. The arc length measured along the MEP is normalized by that from $\psi_{3}$ to $\psi_{2}$, hence $s$ runs from 0 to 1 . In each set of the data, the corresponding value at $\psi_{3}$ has been subtracted to let the curve start from zero. (b) Distributions of order parameter magnitude (left) and current density (right) for a sequence of states labeled along the curve of free energy in (a). In the left panel, the gray scale varies from black for $|\psi|=0$ to white for $|\psi|=1$, and the arrows denote the direction of vortex motion across the strip. Only the segments of noticeable spatial variations are shown for clear illustration.

MEP from $\psi_{3}$ to $\psi_{2}$. The free-energy barrier along this MEP is evaluated to be $\Delta F^{\mathrm{PSV}}=F\left[\psi_{s}^{\mathrm{PSV}}\right]-F_{3}=5.13$. The PSS MEP is also obtained but not depicted here, and the corresponding free-energy barrier is found to be $\Delta F^{\mathrm{PSS}}=F\left[\psi_{s}^{\mathrm{PSS}}\right]-F_{3}$ $=10.9$ (in agreement with the prediction of the LAMH theory), much higher than $\Delta F^{\mathrm{PSV}}=5.13$. Displayed in Fig. 2(b) is a sequence of states along the PSV MEP from $\psi_{3}$ to $\psi_{2}$, showing that a vortex (with clockwise current circulation) is first nucleated at the lower edge $(y=-w / 2)$, then moves across the strip, and is finally annihilated at the upper edge $(y=+w / 2)$. [Because of the mirror symmetry of system with respect to $y=0$, there exists another PSV MEP that involves an antivortex (with counterclockwise current circulation) crossing the strip from the upper to the lower edge. This MEP leads to the same free-energy barrier and has been observed by using a slightly modified initial string.] In particular, state II in Fig. 2(b) is the stationary PSV solution (i.e., the saddle point), in which the position of vortex center is 


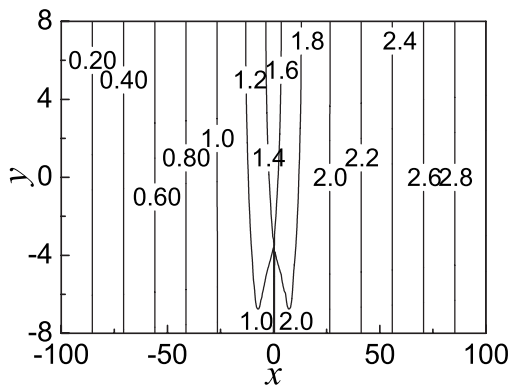

FIG. 3. Contour plot of the phase distribution $\varphi(x, y)$ (in the unit of $2 \pi$ ) for the PSV solution [state II in Fig. 2(b)].

found to be $y_{m} \cong-3.85$, very close to $y_{m}=-3.90$ predicted by the London theory [according to Eq. (A4)]. To understand how the phase slip occurs in $2 \mathrm{D}$ space, we turn to the phase distribution $\phi(x, y)$ of the complex order parameter. Figure 3 shows the contour plot of $\phi(x, y)$ for the PSV solution [state II in Fig. 2(b)], from which the existence of a vortex is clearly observed. This vortex gives rise to a constant difference of $2 \pi$ between the phase variations below and above the vortex core. As a consequence, the phase change accumulated along the $+x$ direction will decrease by $2 \pi$ once the vortex traverses the strip along the $+y$ direction.

Figure 2(a) also shows the variations of the condensation energy and kinetic energy along the PSV MEP. The variation of the condensation energy shows three platforms. The platform " $\mathrm{A}-\mathrm{B}$ " in the middle is of height $\cong 1.56$, corresponding to the MEP segment between states I and III. This platform is produced by the extra condensation energy used in forming a vortex. In particular, "A" and "B" correspond to states I and III, respectively, which are associated with the emergence and disappearance of the vortex. Additional calculations show this extra condensation energy to be only weakly dependent on the external current. However, the kinetic energy barrier $[\cong 3.58$ in Fig. 2(a)] is very sensitive to the external current. Our calculations show that the kinetic energy barrier reaches the maximum value at zero current and decreases with the increasing current. When the current is large enough, the kinetic energy exhibits no barrier but a monotonic decrease along the PSV MEP. That is, the kinetic energy alone actually prefers a vortex to emerge and cross the strip. This is, of course, counterbalanced by the condensation energy barrier associated with the vortex formation. (Otherwise, the metastability of the current-carrying states would be lost.) The total free-energy barrier approaches zero when the critical current density $j_{c}$ is reached. This results in frequent phase slips induced by the numerous vortices easily nucleated at the edges. Our numerical study is not applicable to this extreme regime where phase slips are no longer rare events.

\section{B. Comparison between the Ginzburg-Landau results and the London results}

Figures 4(a) and 4(b) show the free-energy barrier $\Delta F^{\mathrm{PSV}}$ as a function of strip width $w$, evaluated for current densities $j=0.02 j_{c}$ and $0.162 j_{c}$, which are realized in numerical calculations by using a large length $l=256 \pi$ and initial winding
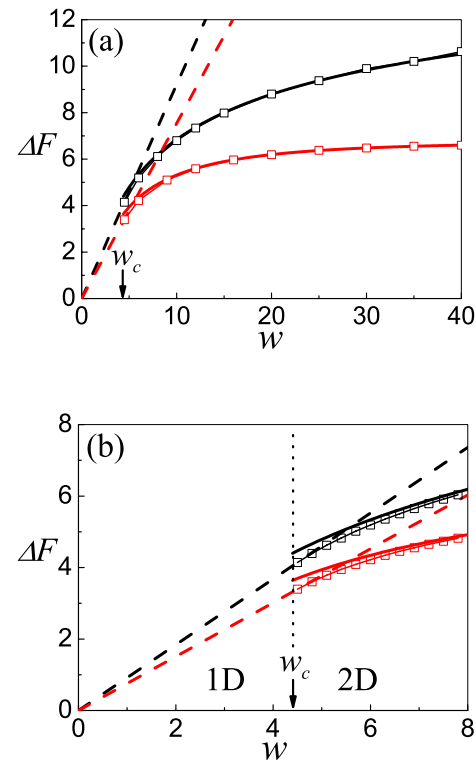

FIG. 4. (Color online) (a) Free-energy barrier plotted as a function of strip width $w$, evaluated for current densities $j=0.02 j_{c}$ (black) and $0.162 j_{c}$ (red). The squares represent $\Delta F^{\mathrm{PSV}}$ for the PSV solution obtained from the GL theory by employing the string method, while the solid lines represent $\Delta F^{\mathrm{PSV}}$ expressed by Eq. (A3), derived from the London theory. The dashed lines represent the free-energy barrier $\Delta F^{\mathrm{PSS}} \propto w$ for the PSS solution, calculated according to the LAMH theory. (b) An enlarged view of (a) for small strip width. A critical width $w_{c}$ is noted to separate the $2 \mathrm{D}$ PSV regime above $w_{c}$ from the 1D PSS regime below $w_{c}$.

numbers $n=1$ (for $j=0.02 j_{c}$ ) and $n=8$ (for $j=0.162 j_{c}$ ). Two sets of data are presented in Fig. 4 for the PSV solution. The squares represent the numerical results obtained from the GL theory by using the string method, while the solid lines represent the predictions of the London theory, expressed by Eq. (A3). Excellent agreement has been achieved for large strip width. However, difference between the two data sets becomes clearly visible as the strip width is reduced. This is expected since in a narrow strip the vortex is too close to the edge to allow a satisfactory description by the London theory. In Fig. 4, there is only one adjustable parameter used to optimize the agreement between the London results and the GL results. This is the vortex core energy $E_{c}$ which appears in Eqs. (A2) and (A3). To optimize the fitting in Fig. 4, two slightly different core energy values are used: $E_{c} \cong 1.27$ for $j=0.02 j_{c}$ and $E_{c} \cong 1.22$ for $j=0.162 j_{c}$. These values are considered to be in reasonable agreement with the condensation energy barrier associated with the formation of a vortex, found to be 1.56 along the PSV MEP shown in Fig. 2. It is also noted that the above fitting values for the core energy are much higher than the usual rough estimation $\left(\pi \xi^{2} d H_{c}^{2} / 8 \pi\right) / G \cong 0.785$ (Refs. 9 and 10 ) but a bit lower than the value $\sim 1.42$ deduced from the London theory in the strong current limit (see Appendix A).

\section{Crossover from two-dimensional strip to one-dimensional wire}

Physically, it is expected that, as the strip width is gradually reduced, it will reach a critical width $w_{c}$ below which 
phase slips can no longer be realized through vortices crossing the strip. Rather, the strip acts effectively as a wire since order parameter variations across the strip are prohibited. In Ref. 30, a minimum strip width has been numerically evaluated for the existence of vortices: $w_{c} \cong 4.4$. We have numerically verified this criterion for thermally activated phase slips using the GL theory. In Fig. 4, the critical width $w_{c}$ is found to be between 4.3 and 4.5 . It is our numerical finding that below the critical width, although order parameter variations along the $y$ direction are still allowed in computation, only those phase slips that occur through the 1D PSS solution are obtained. [That is, by evolving a string toward the stationary MEP, any order parameter variation along the $y$ direction would be gradually removed and a PSS MEP is always reached in the end.] Above $w_{c}$, however, phase slips may occur through either the 1D PSS or the 2D PSV solution. The dominant contribution is made through the saddle point of lower free energy. To compare the variation of $\Delta F^{\mathrm{PSS}}$ with that of $\Delta F^{\mathrm{PSV}}$, we start from the analytical expression derived from the London theory [Eq. (A3)], which shows the free-energy barrier $\Delta F^{\mathrm{PSV}}$ to increase logarithmically with the width $w$ at most: the logarithmic dependence is attained for zero current only, while for finite currents the increase is slower. In fact, $\Delta F^{\mathrm{PSV}}$ approaches a saturation value as $\pi / 2 w k \rightarrow 0$, where $k$ is the wave vector [see Eq. (A3)]. This tendency is seen clearly in Fig. 4(a): the curve for the larger current density shows a much slower rise with $w$. On the other hand, the 1D nature of the PSS solution leads to a linear increase in $\Delta F^{\mathrm{PSS}}$ with $w$, much faster than that of $\Delta F^{\mathrm{PSV}}$. It is therefore inevitable that, for wide strips, $\Delta F^{\mathrm{PSS}}>\Delta F^{\mathrm{PSV}}$, and hence phase slips are dominated by vortices crossing the strip, which are activated with a lower barrier. In particular, Fig. 4(b) shows that $\Delta F^{\mathrm{PSS}}$ surpasses $\Delta F^{\mathrm{PSV}}$ starting from $w=w_{c}$, below which the PSS solution is the only channel for phase slips to occur. Note that around the critical width $w_{c} \cong 4.4$, accurate results can only be obtained from the GL theory because the vortex core is very close to the strip edge and thus the London theory is not reliable. It is also interesting to note that in Fig. 4(b), within numerical error, we have $\Delta F^{\mathrm{PSS}}=\Delta F^{\mathrm{PSV}}$ at the critical width $w_{c}$, below which the PSV solution ceases to exist.

In summary, the critical strip width $w_{c} \cong 4.4$ separates the 1D PSS regime and the 2D PSV regime for thermally activated phase slips. We note that in Refs. 16 and 17, it is argued that the PSS solution is responsible for the formation of resistive state in their samples, typically of width $w \sim 10\left(>w_{c}\right)$ in a wide temperature range below $T_{c}$.

\section{Boundary conditions}

The numerical results presented above are obtained by using superconductor-insulator boundary condition at the strip edges $y= \pm w / 2$. If the superconductor-metal boundary condition is employed, ${ }^{31,32}$ the current-carrying metastable states no longer possess uniform distribution of order parameter along the $y$ direction across the strip because of the suppressed superconductivity near the edges, and consequently numerical evaluation of these states becomes necessary. Even the stationary PSS solutions will show some variation of order parameter along the $y$ direction, and hence their quantitative description goes beyond the 1D LAMH theory. As for the PSV solutions, the London theory is made less accurate by the near-edge variation of order parameter, especially for narrow strips. We want to point out that, for the superconductor-metal boundary condition, the string method has also been employed to numerically calculate the metastable states, PSS solutions (with 2D variations of the order parameter) and PSV solutions, and the corresponding free-energy barriers. Although the numerical results are not presented here, they are found to be in qualitative agreement with the results under the superconductor-insulator boundary condition. Physically, under the superconductor-metal boundary condition, the strip is effectively narrowed by the suppressed order parameter near the geometrical edges. This leads to a larger critical width separating the PSS and PSV regimes, but the physical picture for the crossover from 2D to $1 \mathrm{D}$ behavior remains unchanged.

\section{CONCLUSION}

In the framework of GL theory, we have numerically calculated the saddle points and the corresponding free-energy barriers for thermally activated phase slips in narrow superconducting strips by using the string method. The numerical results show the existence of a critical width. Below the critical width, the strip behaves as a 1D wire with phase slips carried by the PSS solutions. Above the critical width, the strip shows the 2D character, with phase slips realized through the PSV solutions that involve vortex motion across the strip. Therefore, our numerical study based on the GL theory provides a unified description for phase slips in narrow systems ranging from $1 \mathrm{D}$ wires to $2 \mathrm{D}$ strips. For wide strips, good agreement has been obtained with the predictions of the London theory, while for narrow strips, our numerical results represent improvement over the theoretical results.

\section{ACKNOWLEDGMENTS}

This work was partially supported by the Hong Kong RGC under Grants No. HKUST 602904 and No. HKUST 602805 .

\section{APPENDIX A: ANALYTICAL RESULTS FROM THE LONDON THEORY}

This is a brief review for the phase-slip-vortex solution and the corresponding energy barrier described by the London theory. For consistency, we use the same dimensionless quantities defined in Sec. III A. As shown in Fig. 1, we consider the current flowing in the $+x$ direction with a vortex moving in the $+y$ direction. (An extension to an antivortex moving in the $-y$ direction is straight forward based on symmetry operation.) For a 2D strip of a finite width $w$ and an infinite length, if no current is applied, then the self-energy of a vortex at position $y$ (with $-w / 2<y<w / 2)$ can be written as 


$$
F(y)=\pi \ln \left(\frac{2 w}{\pi} \cos \frac{\pi y}{w}\right)+E_{c} .
$$

In the right-hand side of this equation, the first term represents the energy arising from the interaction among the vortex and a series of image vortices, and the second term is the positive vortex core energy which is sometimes ignored. ${ }^{19,33}$ The core energy is included because the condensation energy is underestimated while the kinetic energy (of circulating current within the vortex core of radius $\sim 1$ ) is overestimated by ignoring the core structure characterized by the suppressed order parameter. Equation (A1) diverges at $y= \pm w / 2$ and is considered not valid within a distance $\sim 1$ from the edges. This is expected because the size of vortex core has not been taken into account in Eq. (A1)

In the presence of external current, with the additional current-vortex interaction, the energy becomes ${ }^{33}$

$$
F(y)=\pi \ln \left(\frac{2 w}{\pi} \cos \frac{\pi y}{w}\right)-2 \pi k(y+w / 2)+E_{c},
$$

where $k$ is the wave vector in the $+x$ direction. The energy barrier for a single vortex crossing the strip can be found by locating the maximum of $F(y)$,

$$
\Delta F^{\mathrm{PSV}}=\pi\left[\ln \frac{2 w}{\pi \sqrt{1+(2 w k / \pi)^{2}}}-\frac{2 w k}{\pi} \tan ^{-1}\left(\frac{\pi}{2 w k}\right)\right]+E_{c},
$$

which occurs at

$$
y_{m}=\frac{w}{\pi} \tan ^{-1}\left(\frac{\pi}{2 w k}\right)-\frac{w}{2},
$$

corresponding to the position of vortex center in the PSV solution.

The core energy $E_{c}$ is usually treated as an adjustable parameter. To have an estimate of $E_{c}$, we take $k=k_{c}=1 / \sqrt{3}$, which is the critical wave vector for vanishing metastability, and then require $\Delta F^{\mathrm{PSV}}=0$. This yields $E_{c} \cong 1.42$. In this estimation, the distance of the vortex from the lower edge $y=-w / 2,\left|y_{m}+w / 2\right|=1 / 2 k_{c} \cong 0.866$, is already so small that the London theory is no longer considered accurate. However, we still find this estimate to be very close to our fitting values $\left(E_{c} \cong 1.27\right.$ and 1.22 ; see Sec. IV B) used in matching the GL results for $\Delta F^{\mathrm{PSV}}$.

\section{APPENDIX B: STRING METHOD}

To outline the string method, ${ }^{20-22}$ we consider a system governed by the overdamped Langevin equation,

$$
\gamma \dot{q}_{i}=-\nabla_{i} V(\mathbf{q})+\zeta_{i}(t)
$$

where $\gamma$ is the damping coefficient, $\mathbf{q}$ denotes the generalized coordinates $\left\{q_{i}\right\}, \dot{q}_{i} \equiv \partial q_{i} / \partial t, \nabla_{i} \equiv \partial / \partial q_{i}$, and $\zeta_{i}(t)$ is a white noise satisfying $\left\langle\zeta_{i}(t) \zeta_{j}\left(t^{\prime}\right)\right\rangle=2 \gamma k_{B} T \delta_{i j} \delta\left(t-t^{\prime}\right)$. Metastable and stable states are located in configuration space as the minima of the potential $V(\mathbf{q})$. Let $\mathbf{q}_{A}$ and $\mathbf{q}_{B}$ represent the two minima of $V(\mathbf{q})$. In terms of the topography of $V(\mathbf{q})$, the most probable fluctuation that can carry the system from $\mathbf{q}_{A}$ to $\mathbf{q}_{B}$ (or $\mathbf{q}_{B}$ to $\mathbf{q}_{A}$ ) corresponds to the lowest intervening saddle point between these two minima. The minimal energy path (MEP) is defined as a smooth curve $\widetilde{\mathbf{q}}(s)$ in configuration space. With intrinsic parametrization such as arc length $s$, the MEP connects $\mathbf{q}_{A}$ and $\mathbf{q}_{B}$ and satisfies

$$
(\nabla V)^{\perp}(\widetilde{\mathbf{q}})=0,
$$

where $(\nabla V)^{\perp}$ denotes the component of $\nabla V$ locally normal to the path $\widetilde{\mathbf{q}}(s)$. Physically, the MEP is the most probable pathway for thermally activated transitions from $\mathbf{q}_{A}$ to $\mathbf{q}_{B}$ and vice versa. To numerically locate the MEP in configuration space, a string $\mathbf{q}(s)$ connecting $\mathbf{q}_{A}$ and $\mathbf{q}_{B}$ is parametrized by arc length $s$ and evolved according to

$$
\dot{\mathbf{q}}=-(\nabla V)^{\perp}(\mathbf{q}) \text {. }
$$

A reparametrization has to be applied once in a while to enforce accurate parametrization by $s$. The stationary solution of Eq. (B3) satisfies Eq. (B2) by which the MEP is defined. Once the MEP is determined, the saddle point and the corresponding energy barrier can be obtained by locating the point of maximum potential along the MEP.

\footnotetext{
*Author to whom correspondence should be addressed; maqian@ust.hk

${ }^{1}$ W. A. Little, Phys. Rev. 156, 396 (1967).

${ }^{2}$ J. S. Langer and V. Ambegaokar, Phys. Rev. 164, 498 (1967).

${ }^{3}$ D. McCumber and B. Halperin, Phys. Rev. B 1, 1054 (1970).

${ }^{4}$ For a review, see, W. J. Skocpol and M. Tinkham, Rep. Prog. Phys. 38, 1049 (1975).

${ }^{5}$ J. M. Kosterlitz and D. J. Thouless, J. Phys. C 6, 1181 (1973).

${ }^{6}$ M. R. Beasley, J. E. Mooij, and T. P. Orlando, Phys. Rev. Lett. 42, 1165 (1979).

${ }^{7}$ S. Doniach and B. A. Huberman, Phys. Rev. Lett. 42, 1169 (1979)

${ }^{8}$ B. I. Halperin and D. R. Nelson, J. Low Temp. Phys. 36, 599 (1979).
}

${ }^{9}$ L. A. Turkevich, J. Phys. C 12, L385 (1979).

${ }^{10}$ P. Minnhagen, Phys. Rev. B 24, 6758 (1981).

${ }^{11}$ A. M. Kadin, K. Epstein, and A. M. Goldman, Phys. Rev. B 27, 6691 (1983).

${ }^{12}$ A. Verevkin, J. Zhang, R. Sobolewski, A. Lipatov, O. Okunev, G. Chulkova, A. Korneev, K. Smirnov, G. N. Goltsman, and A. Semenov, Appl. Phys. Lett. 80, 4687 (2002).

${ }^{13}$ J. Kitaygorsky, J. Zhang, A. Verevkin, A. Sergeev, A. Korneev, V. Matvienko, P. Kouminov, B. Voronov, G. Gol'tsman, and R. Sobolewski, IEEE Trans. Appl. Supercond. 15, 545 (2005).

${ }^{14}$ A. Engel, A. D. Semenov, H.-W. Hbers, K. Il'in, and M. Siegel, Physica C 444, 12 (2006).

${ }^{15}$ J. Kitaygorsky, I. Komissarov, A. Jukna, D. Pan, O. Minaeva, N. Kaurova, A. Divochiy, A. Korneev, M. Tarkhov, B. Voronov, I. 
Milostnaya, G. Gol'tsman, and Roman R. Sobolewski, IEEE Trans. Appl. Supercond. 17, 275 (2007).

${ }^{16}$ M. Bell, A. Sergeev, V. Mitin, J. Bird, A. Verevkin, and G. Gol'tsman, Phys. Rev. B 76, 094521 (2007).

${ }^{17}$ M. Bell, N. Kaurova, A. Divochiy, G. Gol'tsman, J. Bird, A. Sergeev, and A. Verevkin, IEEE Trans. Appl. Supercond. 17, 267 (2007).

${ }^{18}$ A. D. Semenov, Physica C 416, 85 (2004).

${ }^{19}$ V. G. Kogan, Phys. Rev. B 49, 15874 (1994).

${ }^{20}$ W. E, W. Ren, and E. Vanden-Eijnden, Phys. Rev. B 66, 052301 (2002).

${ }^{21}$ W. E, W. Ren, and E. Vanden-Eijnden, J. Appl. Phys. 93, 2275 (2003)

${ }^{22}$ W. E, W. Ren, and E. Vanden-Eijnden, J. Chem. Phys. 126, 164103 (2007).

${ }^{23}$ T. Qian, W. Ren, and P. Sheng, Phys. Rev. B 72, 014512 (2005).

${ }^{24}$ C. Qiu, T. Qian, and W. Ren, Phys. Rev. B 77, 104516 (2008).
${ }^{25}$ V. Ambegaokar, B. I. Halperin, D. R. Nelson, and E. D. Siggia, Phys. Rev. B 21, 1806 (1980).

${ }^{26}$ I. Aranson, L. Kramer, and A. Weber, J. Low Temp. Phys. 89, 859 (1992).

${ }^{27}$ I. Aranson, M. Gitterman, and B. Y. Shapiro, J. Low Temp. Phys. 97, 215 (1994).

${ }^{28}$ J. Pearl, Appl. Phys. Lett. 5, 65 (1964).

${ }^{29}$ G. Henkelman, B. P. Uberuaga, and H. Jonsson, J. Chem. Phys. 113, 9901 (2000).

${ }^{30}$ K. K. Likharev, Rev. Mod. Phys. 51, 101 (1979).

${ }^{31}$ M. Tinkham, Introduction to Superconductivity (McGraw-Hill, New York, 1995).

${ }^{32}$ A. D. Hernandez and D. Dominguez, Phys. Rev. B 65, 144529 (2002).

${ }^{33}$ F. Tafuri, J. R. Kirtley, D. Born, D. Stornaiuolo, P. G. Medaglia, P. Orgiani, G. Balestrino, and V. G. Kogan, Europhys. Lett. 73, 948 (2006) 